\title{
Synthesis and Density Functional Theory Analysis of Pyrazole Integrated Pyrimidinetrione
}

\author{
Shunmugam Iniyaval, Krishnaraj Padmavathy, Ramar Sivaramakarthikeyan, \\ Chennan Ramalingan
}

\begin{abstract}
A pyrazole integrated pyrimidinetrione 6 has been synthesized and its structure has been established employing physical and analytical techniques such as IR and NMR spectroscopy. Further, density functional theory studies such as geometry optimization, FMOs, MEP, Mulliken population and $N L O$ analyses of the synthesized compound 6 have been carried out using DFT - B3LYP [6-311++G(d,p)] method and the results obtained have been discussed.
\end{abstract}

Keywords: Pyrazole, Barbituric acid, Density Functional Theory

\section{INTRODUCTION}

Pyrazole is a heterocyclic compound that has two adjacent nitrogen atoms with three carbon atoms in a five membered ring. Infact, Pyrazoles is the key unit present in the skeletal ring of hemoglobin. Pyrazoles hold stream of biological potencies such as antimicrobial, anti-inflammatory, antifungal, antiviral, and antitumor activities [1-5] due to its better ADME properties. In recent days, pyrazole has engrossed the interest of pharmacological industries as a leading compound in numerous drug discovery and development, particularly, in the field of cancer therapy [6]. The compound 7 - ( 1 - (m-chlorophenyl)-3-(pmethoxyphenyl)-1H-pyrazol-4-yl)-5-oxo-2-p-tolyltriazolo[1, 5-a]-pyridine-6,8-dicarbonitrile was reported to display anticancer potency against SW-620 cell line (colon) with GI50 of $0.52 \mu \mathrm{M}$, also found to be active against cell lines of renal cancer viz., RXF 393 and A498 with GI50 of 0.86 and $0.58 \mu \mathrm{M}$, respectively.[7] Besides their varied biological activities, they also find applications in agrochemicals, and serve as luminophores and fluorophores [8-15]. Certain

Revised Manuscript Received on December 15, 2019.

* Correspondence Author

Shunmugam Iniyaval, Department of Chemistry, School of Advanced Sciences, Kalaslaingam Academy of Research and Education (Deemed to be University), Krishnankoil, 626 126, Tamilnadu, India. Email: iniyaval.s@klu.ac.in

Krishnaraj Padmavathy, Department of Chemistry, School of Advanced Sciences, Kalasalingam Academy of Research and Education (Deemed to be University), Krishnankoil, 626 126, Tamilnadu, India. Email: ovshada@gmail.com

Ramar Sivaramakarthikeyan, Department of Chemistry, School of Advanced Sciences, Kalasalingam Academy of Research and Education (Deemed to be University), Krishnankoil, 626 126, Tamilnadu, India. Email: sivaram.ramar@gmail.com

*Chennan Ramalingan, Department of Chemistry, School of Advanced Sciences, Kalasalingam Academy of Research and Education (Deemed to be University), Krishnankoil, 626 126, Tamilnadu, India. Email: ramalinganc@gmail.com pyrazole nopionone derivatives were reported to emit strong blue fluorescence in ethanol and were found to detect the copper sulfate pentahydrate ( $\geq 99 \%$ ) content $(100.57 \%$ ) with a RSD of $1.98 \%$. [16]

On the other hand, barbiturates are closed chain ureic compounds used in treatment of sleep disorders and for the management of epileptic seizures. Specifically, oxybarbiturates, continues to be the selected drugs in the treatment of insomnia and in some types of epilepsy. Sodium pentothal has been used as an intravenous anesthetic with fast recovery and no side effects. [17] From the literature, barbiturates demonstrate a various biological/ pharmacological applications including anesthesia, anticonvulsant, sedation, anxiolytic, hypnosis, antioxidant, antifungal, antibacterial, anticancer, anti-osteroporosis and inhibition of aminotransferase, alpha-glucosidase, diaminopimelate and tyrosine. [18-26] Apart from biological applications of barbiturates, they are reported to show NLO properties. [27] Chitosan-barbiturate ( $\mathrm{Ch}-\mathrm{Ba}$ ) derivatives were stated to be used in the preparation of gel polymer electrolytes as a host polymer [28].

Based on the diverse range of applications revealed by pyrazole and barbituric acid, we proposed to synthesize a molecule by tailoring both heterocyclic structural motifs. The synthesized compound was then confirmed using IR and NMR techniques. Further, various theoretical studies have been performed utilizing DFT method.

\section{EXPERIMENTAL SECTION}

\section{A. Materials and methods}

All the solvents and reagents were either purified by adopting general purification methods or analytical reagent grade were utilized. The reported MPs were measured in open capillaries. Infrared (FT-IR) spectrum ( $\mathrm{KBr}$ pellet) was recorded on Shimadzu spectrophotometer (IR Tracer-100). NMR spectra were recorded using Bruker spectrometer with DMSO- $\mathrm{d}_{6}$ as a solvent.

\section{B. Synthesis of carbaldehyde $4^{\text {[29] }}$}

A mixture of ketone $1(0.01 \mathrm{~mol})$ and hydrazine 2 (0.01 $\mathrm{mol})$ in glacial acetic acid $(10 \mathrm{~mL})$ was heated for 30 minutes. It was filtered after cooling. The solid thus obtained was rinsed with dilute hydrochloric acid solution. Then the solid was purified through recrystallization using ethanol.

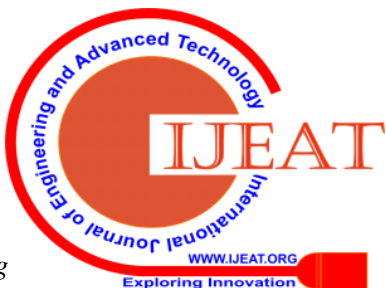


The pure hydrazone (3) thus obtained was treated with Vilsmeier-Haack reagent [DMF $\left.(10 \mathrm{ml})-\mathrm{POCl}_{3}(0.03 \mathrm{~mol})\right]$ at $0{ }^{\circ} \mathrm{C}$ and it was stirred at $70-80{ }^{\circ} \mathrm{C}$ for $6 \mathrm{~h}$. It was then allowed to attain ambient temperature and poured in to water. The solid was filtered, after neutralization with $\mathrm{NaHCO}_{3}$ (saturated), and recrystallized, after washed with water and dried, to afford pure product 4 in $78 \%$ yield.

\section{Synthesis of pyrazolylpyrimidinetrione 6 .}

A 1:1 mixture of $4(0.3 \mathrm{~g}, 0.91 \mathrm{mmol})$ and barbituric acid

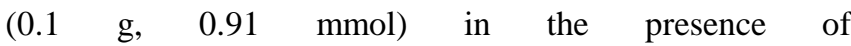
benzyltriethylammonium chloride $(0.01 \mathrm{~g}, 5 \mathrm{~mol} \%)$ in ethylene glycol $(10 \mathrm{ml})$ was heated at $100{ }^{\circ} \mathrm{C}$ for $4 \mathrm{~h}$. After completion, it was cooled, poured into water and the solid thus obtained was washed with hot ethanol, after filtration, to furnish the target $\mathbf{6}$ in a pure form.

Yield: $0.29 \mathrm{~g}$ (73\%), MP. $252-254{ }^{\circ} \mathrm{C}$. IR $\left(\mathrm{KBr}, \mathrm{cm}^{-1}\right)$ : 3342.6, 3174.8, 3057.2, 2831.5, 1666.5, 1566.2, 1394.5, $1342.5,1211.3,854.9,796.6,719.5,686.7,584.3,511.1 ;{ }^{1} \mathrm{H}$ NMR(400 MHz, DMSO-d 6 ): $\delta 11.37$ (s, 1H), 11.34, (s, 1H), $9.79(\mathrm{~s}, 1 \mathrm{H}), 8.13(\mathrm{~s}, 1 \mathrm{H}), 7.94(\mathrm{~d}, \mathrm{~J}=7.60 \mathrm{~Hz}, 2 \mathrm{H}), 7.85$ (s, $1 \mathrm{H}), 7.79(\mathrm{~d}, \mathrm{~J}=7.60 \mathrm{~Hz}, 1 \mathrm{H}), 7.61-7.55(\mathrm{~m}, 4 \mathrm{H}), 7.50-7.47$ $(\mathrm{m}, 1 \mathrm{H}),{ }^{13} \mathrm{C}$ NMR $\left(100 \mathrm{MHz}, \mathrm{DMSO}-\mathrm{d}_{6}\right): \delta 164.0,163.1$, 156.7, 150.7,143.3, 139.0, 135.1, 133.6, 132.7, 132.3, 131.5, $130.4,129.1,128.7,122.6,120.2,115.7,115.4$

\section{Computational procedure}

Computational studies were executed with the help of Gaussian 09W [30] software package. Using Berny method [31] with hybrid function B3LYP [6-311++G(d,p)], density function theory was adopted for all theoretical calculations. The structural and energy parameters such as optimized molecular geometry, molecular orbital descriptions HOMO, LUMO, electron density, MEP and NLO properties of $\mathbf{6}$ were obtained from the computational calculations.

\section{RESULT AND DISCUSSION}

\section{A. Synthesis}

The target molecule $\mathbf{6}$ has been synthesized through multi-step synthesis and the schematic representation for the synthesis is provided in Scheme 1.

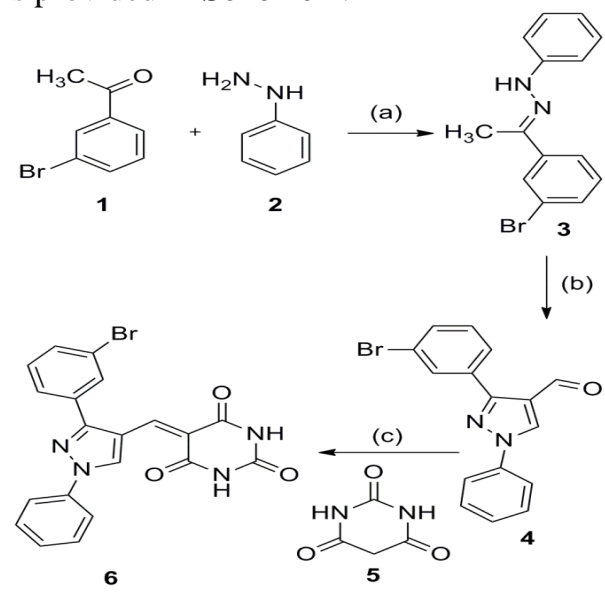

Scheme 1: Synthesis of target molecule 6. Reagents and conditions: (a) Glacial acetic acid, reflux, $30 \mathrm{~min}$; (b) $\mathrm{DMF}^{-\mathrm{POCl}_{3}, 70-80}{ }^{\circ} \mathrm{C}, 6 \mathrm{~h}$; (c) Benzyltriethylammonium chloride, ethylene glycol, $100{ }^{\circ} \mathrm{C}, 4 \mathrm{~h}$.
The key intermediate, carbaldehyde $\mathbf{4}$ has been synthesized from the ketone $\mathbf{1}$ by condensation with hydrazine $\mathbf{2}$ followed by cyclization and formylation reactions. The target molecule $\mathbf{6}$ has been eventually synthesized by the condensation of $\mathbf{4}$ with $\mathbf{5}$ in the presence of phase transfer catalyst. The structure of the target $\mathbf{6}$ has been established based on physical and spectral data.
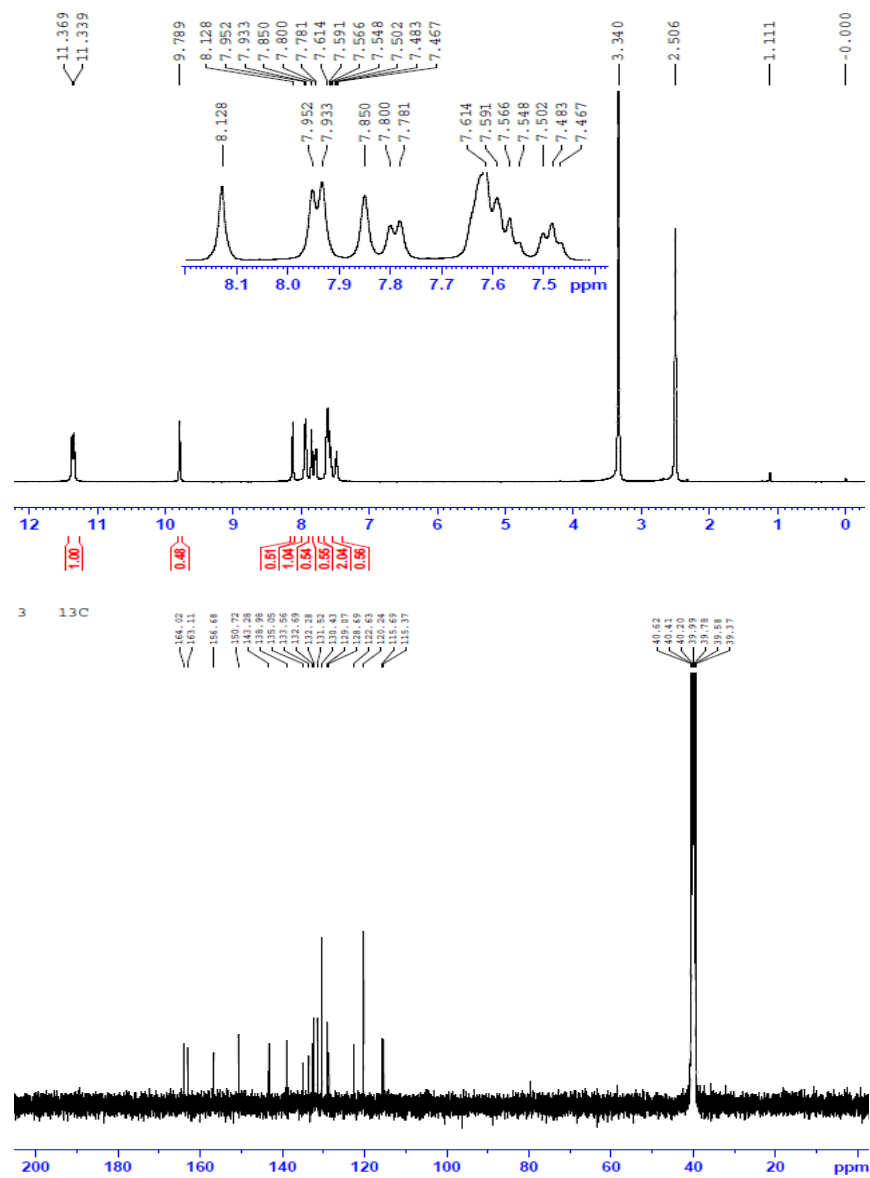

Fig. 1: ${ }^{1} \mathrm{H}$ and ${ }^{13} \mathrm{C}$ NMR spectrum of the title molecule 6 .

\section{B. Optimized geometry}

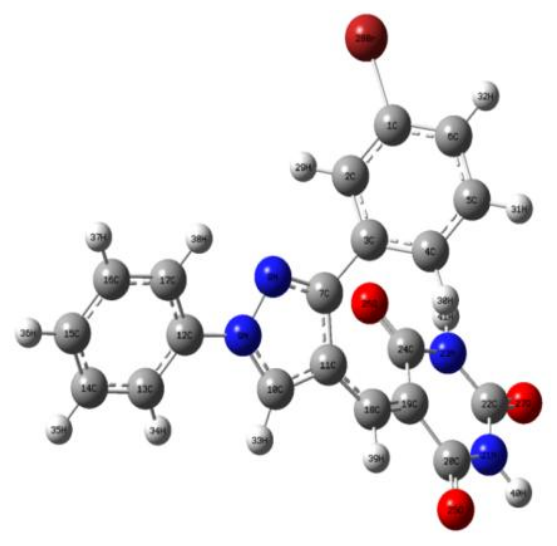

Fig. 2: Optimized geometry of the target molecule 6. 
The optimized structure of pyrazole based pyrimidinetrione 6 was accomplished and it is provided in Fig. 2. The parameters viz., bond length, bond angle and dihedral angle values are listed in Tables I \& II. The lengths of C-N bonds of pyrazole ring are found to be $1.3244 \AA$ (C7-N8) and $1.3499 \AA$ (C10-N9) which lie intermediary between the C-N single $(1.443 \AA)$ and double $(1.269 \AA)$ bonds signifying electron delocalization. The bond angles of C11-C18-C19, N8-N9-C12 and C3-C7-N8 are 132.5, 120.09 and 118.83 degrees, respectively.

Table-I: Bond length of the title compound 6.

\begin{tabular}{llll}
\hline Bondlength & $(\AA)$ & Bondlength & $(\AA)$ \\
\hline C1-C2 & 1.3873 & C13-C14 & 1.3916 \\
C1-C6 & 1.3916 & C13-H34 & 1.0828 \\
C1-Br28 & 1.92 & C14-C15 & 1.3928 \\
C2-C3 & 1.4002 & C14-H35 & 1.0837 \\
C2-H29 & 1.0807 & C15-C16 & 1.3938 \\
C3-C4 & 1.3986 & C15-H36 & 1.0835 \\
C3-C7 & 1.4736 & C16-C17 & 1.3905 \\
C4-C5 & 1.391 & C16-H37 & 1.0837 \\
C4-H30 & 1.083 & C17-H38 & 1.0809 \\
C5-C6 & 1.3929 & C18-19 & 1.3597 \\
C5-H31 & 1.0841 & C18-H39 & 1.0889 \\
C6-H32 & 1.082 & C19-C20 & 1.4901 \\
C7-N8 & 1.3244 & C19-C24 & 1.4792 \\
C7-C11 & 1.4407 & C20-N21 & 1.3911 \\
N8-N9 & 1.3592 & C20-O25 & 1.2152 \\
N9-C10 & 1.3499 & N21-C22 & 1.3894 \\
N9-C12 & 1.424 & N21-H40 & 1.0116 \\
C10-C11 & 1.3912 & C22-N23 & 1.3861 \\
C10-H33 & 1.0775 & C22-O27 & 1.2082 \\
C11-C18 & 1.4403 & N23-C24 & 1.398 \\
C12-C13 & 1.3959 & N23-H41 & 1.0118 \\
C12-C17 & 1.3957 & C24-O26 & 1.2127 \\
\hline
\end{tabular}

Table-II: Bond angle of the title compound 6.

\begin{tabular}{llll}
\hline Bond angle & $\left(^{\circ}\right)$ & Bond angle & $\left(^{\circ}\right)$ \\
\hline C2-C1-C6 & 121.7229 & C12-C13-H34 & 120.6269 \\
C2-C1-Br28 & 119.1412 & C14-C13-H34 & 119.8769 \\
C6-C1-Br28 & 119.1358 & C13-C14-C15 & 120.4241 \\
C1-C2-C3 & 119.3951 & C13-C14-H35 & 119.3532 \\
C1-C2-H29 & 120.9332 & C15-C14-H35 & 120.2161 \\
C3-C2-H29 & 119.6689 & C14-C15-C16 & 119.6018 \\
C2-C3-C4 & 119.3875 & C14-C15-H36 & 120.1573 \\
C2-C3-C7 & 119.4326 & C16-C15-H36 & 120.2394 \\
C4-C3-C7 & 121.1079 & C15-C16-C17 & 120.6258 \\
C3-C4-C5 & 120.2787 & C15-C16-H37 & 120.0979 \\
C3-C4-H30 & 119.9216 & C17-C16-H37 & 119.2755 \\
C5-C4-H30 & 119.7924 & C12-C17-C16 & 119.3113 \\
C4-C5-C6 & 120.6644 & C12-C17-H38 & 119.1167 \\
C4-C5-H31 & 119.9265 & C16-C17-H38 & 121.5716 \\
C6-C5-H31 & 119.4069 & C11-C18-C19 & 132.5331 \\
C1-C6-C5 & 118.5463 & C11-C18-H39 & 114.6545 \\
C1-C6-H32 & 120.5303 & C19-C18-H39 & 112.7892 \\
C5-C6-H32 & 120.9228 & C18-C19-C20 & 115.7784 \\
C3-C7-N8 & 118.8295 & C18-C19-C24 & 124.1685 \\
C3-C7-C11 & 130.3083 & C20-C19-C24 & 119.7214 \\
N8-C7-C11 & 110.5572 & C19-C20-N21 & 115.2755 \\
C7-N8-N9 & 106.3386 & C19-C20-O25 & 124.4685 \\
N8-N9-C10 & 111.5514 & N21-C20-O25 & 120.2521 \\
N8-N9-C12 & 120.0933 & C20-N21-C22 & 127.7937
\end{tabular}

\begin{tabular}{llll} 
C10-N9-C12 & 128.333 & C20-N21-H40 & 116.5443 \\
N9-C10-C11 & 107.6691 & C22-N21-H40 & 115.658 \\
N9-C10-H33 & 122.4667 & N21-C22-N23 & 113.8915 \\
C11-C10-H33 & 129.8495 & N21-C22-O27 & 122.8782 \\
C7-C11-C10 & 103.7687 & N23-C22-O27 & 123.229 \\
C7-C11-C18 & 134.5362 & C22-N23-C24 & 128.2512 \\
C10-C11-C18 & 121.6852 & C22-N23-H41 & 115.6256 \\
N9-C12-C13 & 120.2143 & C24-N23-H41 & 116.0421 \\
N9-C12-C17 & 119.2356 & C19-C24-N23 & 114.8567 \\
C13-C12-C17 & 120.5501 & C19-C24-O26 & 125.3186 \\
C12-C13-C14 & 119.4772 & N23-C24-O26 & 119.7927 \\
\hline
\end{tabular}

\section{HOMO- LUMO analysis}

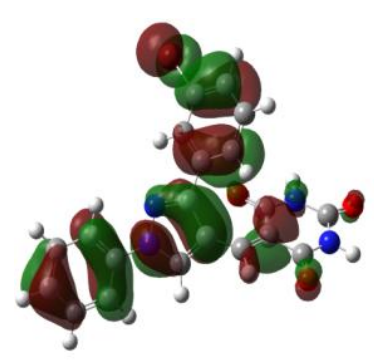

HOMO

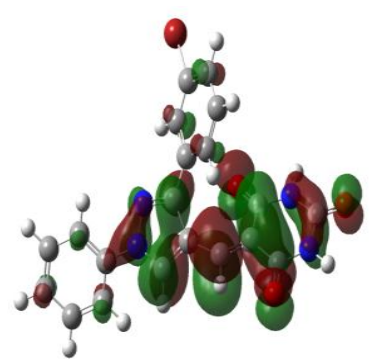

LUMO
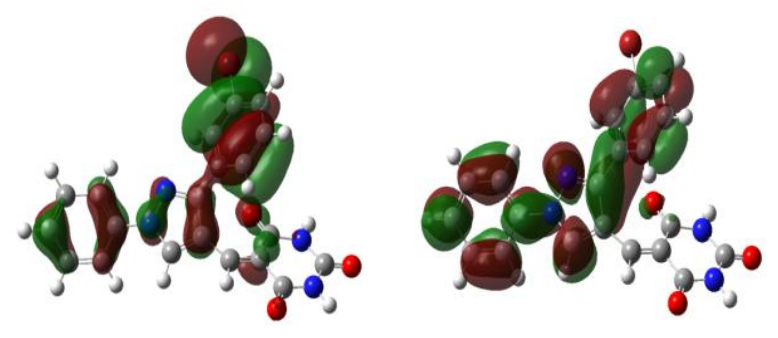

HOMO-1

LUMO+1

The kinetic stability of a molecule could be predicted from the energies of frontier molecular orbitals. When the difference in energy between FMOs orbitals are greater, more stable will be the compound and the reverse will be for smaller energy difference. The FMO plots of the molecule 6 are shown in Fig 3. The energies of the compound 6 are furnished in Table III. HOMO orbitals are highly localized at the two phenyl rings flanked at C7 and N7 of pyrazole moiety whereas the LUMO orbitals are chiefly localized on pyrazole and barbituric acid moiety. The energy gap between HOMO and LUMO was found to be $3.69 \mathrm{eV}$ which is small enough for electron transfer to take place from phenyl rings flanked with pyrazole moiety to the barbituric acid scaffold.

\section{Table- III: HOMO-LUMO energies of molecule 6}

\begin{tabular}{ll}
\hline Parameters $(\mathrm{eV})$ & $\mathrm{eV}$ \\
\hline HOMO & -6.53145 \\
LUMO & -2.84627 \\
$\Delta \mathrm{E}$ & 3.68518 \\
HOMO-1 & -6.86615 \\
LUMO+1 & -1.49959 \\
$\Delta \mathrm{E} 1$ & 5.36656 \\
\hline
\end{tabular}




\section{Synthesis and Density Functional Theory Analysis of Pyrazole Integrated Pyrimidinetrione}

\section{Molecular electrostatic potential analysis}

MEP study of the synthesized compound $\mathbf{6}$ has been carried out by B3LYP [6-311++G(d,p)]. In general, the MEP study aids in prediction of reactive sites of the molecule, intermolecular association and physiochemical affairs. Pictorial representation shown in Fig $\mathbf{4}$ clearly indicated the electron density and MEP of the molecule where the dark blue colour indicates the electron poor centre and red colour indicates the electron rich centre. Negative province is exhibited on the oxygen atom $(\mathrm{O} 25, \mathrm{O} 26$, and $\mathrm{O} 27)$ of the barbituric acid scaffold.

The contour map of electrostatic potential concludes the different positive and negative potential sites of the molecule in agreement with the surface map of total electron density.

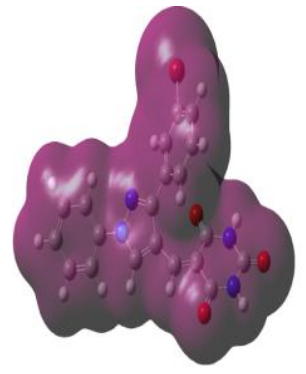

ED

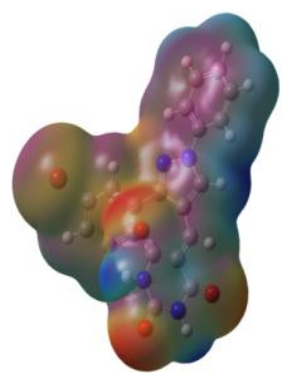

MEP

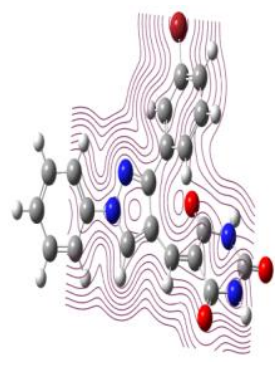

ED with contour

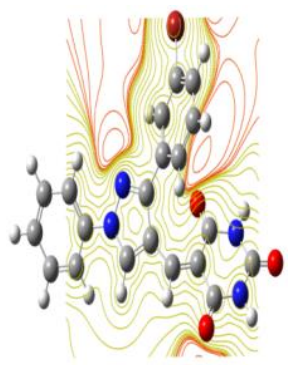

MEP with contour
Fig. 4: Electron density and MEP with their contour of compound 6

\section{E. Mulliken population analysis}

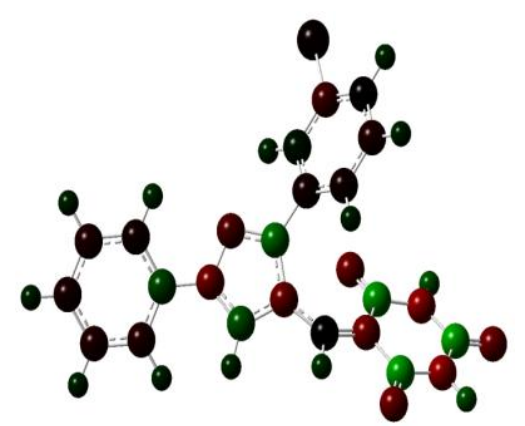

Fig. 5: Mulliken charge distribution of the compound 6
The analysis of Mulliken population of 6 was computed with $6-311++G(d, p)$ method, which can be used as a tool for estimation of partial atomic charges. The N21 atom of the barbituric acid moiety shows elevated negative charge whereas the $\mathrm{C} 22$ atom of the same displayed highest positive charge as shown in Fig. 5 \& 6.

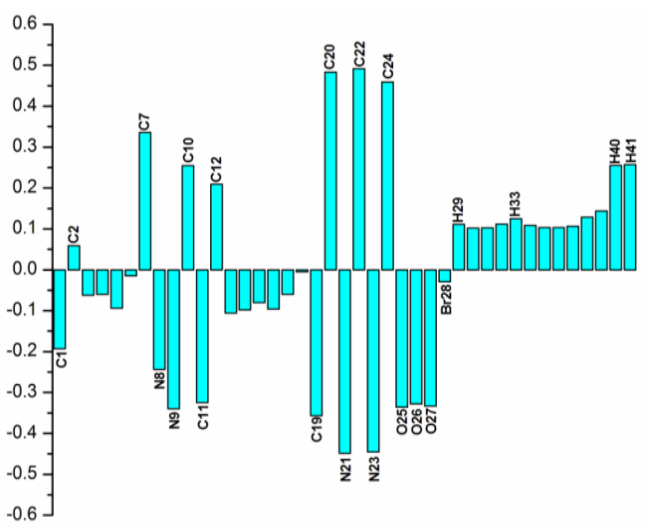

Fig. 6: Mulliken charge distribution chart of the compound 6

To determine the NLO behaviour of the system, the values of polarizability, hyperpolarizability and dipole moment have to be explored. Using B3LYP/ 6-311++G(d,p) basis set, the title compound was computed and the polarizability including hyperpolarizability and dipole moment are evaluated. Its computed values are depicted in Table IV.

Table- IV: NLO properties of title compound

\begin{tabular}{ll}
\hline Parameters & $\mathrm{B} 3 \mathrm{LYP} / 6-311++\mathrm{G}(\mathrm{d}, \mathrm{p})$ \\
\hline Dipole moment & -4.2906 \\
$\mu_{\mathrm{x}}$ & -2.1717 \\
$\mu_{\mathrm{y}}$ & 0.9558 \\
$\mu_{\mathrm{z}}$ & 4.9030 \\
$\mu(\mathrm{D})$ & \\
Polarizability & -159.5937 \\
$\alpha_{\mathrm{xx}}$ & -175.1945 \\
$\alpha_{\mathrm{yy}}$ & -174.0380 \\
$\alpha_{\mathrm{zz}}$ & $4.1027 \times 10^{-23}$ \\
$\alpha_{\mathrm{total}}$ & -169.6087 \\
$\alpha_{0}$ & \\
Hyperpolarizability & -218.0925 \\
$\beta_{\mathrm{xxx}}$ & 46.3732 \\
$\beta_{\mathrm{xxy}}$ & -22.2389 \\
$\beta_{\mathrm{xyy}}$ & 118.7497 \\
$\beta_{\mathrm{yyy}}$ & 17.7739 \\
$\beta_{\mathrm{xxz}}$ & -10.5172 \\
$\beta_{\mathrm{xyz}}$ & -38.1460 \\
$\beta_{\mathrm{yyz}}$ & 35.1716 \\
$\beta_{\mathrm{xzz}}$ & 56.6708 \\
$\beta_{\mathrm{yzz}}$ & -26.3100 \\
$\beta_{\mathrm{zzz}}$ & $2.6412 \times 10^{-30}$ \\
$\beta_{0}$ &
\end{tabular}

The result reveals that the first order hyperpolarizability and dipole moment values are in higher range than urea $(\beta \mathrm{o}=$ $\left.0.37 \times 10^{-30} \mathrm{esu}, \mu(\mathrm{D})=1.3732 \mathrm{D}\right)[32]$. 
The higher hyperpolarizability exhibited by the molecule 6 when compared to urea implies that the molecule could serve as effective NLO material.

\section{CONCLUSION}

In conclusion, successfully synthesized and characterised the target compound $\mathbf{6}$ by hybridisation of heterocyclic pyrazole with pyrimidine trione. Further the structure of $\mathbf{6}$ was optimised, HOMO-LUMO, MEP, NLO properties were analysed. Specifically the first order hyperpolaraizability value is found to be seven fold higher than urea thus the molecule 6 shows NLO characterisation. Besides, the electronic / FMO properties / structural motifs imply that the molecule could serve as effective intermediate for the construction of biopertinent molecules.

\section{ACKNOWLEDGMENT}

Financial assistance provided by the Indian Council of Medical research (No.58/16/2013BMS), New Delhi, India is gratefully acknowledged.

\section{REFERENCES}

1. A.K.Tewari, V.P. Singh, P. Yadav, G. Gupta, A. Singh, R. K. Goel, P. Shinde, C. G. Mohan, "Synthesis, biological evaluation and molecular modeling study of pyrazole derivatives as selective COX-2 inhibitors and anti-inflammatory agents", Bioorg.Chem, vol. 56, pp. 8-15, 2014.

2. S. Ambedkar, V. Padmini, N. Bhuvanesh, "A green and efficient protocol for the synthesis of dihydropyrano[2,3-c]pyrazole derivatives via a one-pot, four component reaction by grinding method", $J$. Adv Res, vol. 6, pp. 975-985, 2015

3. S. Mert, R. Kasimogullari, T. Ica, F. Colak, A. Altun, S. Ok, "Synthesis, structure-activity relationships, and in vitro antibacterial and antifungal activity evaluations of novel pyrazole carboxylic and dicarboxylic acid derivatives", Eur J Med Chem, vol. 78, pp. 86-96, 2014.

4. P. C Iyer, J. Zhao, L.A Emert-Sedlak, K.K Moore, T.E Smithgall, B.W Day, "Synthesis and structure-activity analysis of diphenylpyrazolodiazene inhibitors of the HIV-1 Nef virulence factor", Bioorg Med Chem Lett, vol. 24, pp. 1702-1706, 2014.

5. R.M Mohareb, A.E.M Abdallah, M.A Abdelaziz, "New approaches for the synthesis of pyrazole, thiophene, thieno[2,3-b]pyridine, and thiazole derivatives together with their anti-tumor evaluations", Med Chem Res, vol. 23, pp. 564-579, 2014.

6. S. Kumari, S. Paliwal, R. Chauhan, "Synthesis of Pyrazole Derivatives Possessing Anticancer Activity: Current Status", Synthetic Communications, vol. 44, pp. 1521-1578, 2014.

7. M. M. F. Ismail, N. M. Khalifa, H. H. Fahmy, H. M. EL-Sahrawy, E. S. Nossier, "Anticancer evaluation of novel 1,3,4 - trisubstituted pyrazole candidates bearing different nitrogenous heterocyclic Moieties”, Biomedical Research, vol. 27, pp. 1087-1093,2016.

8. S. Fustero, M. Sanchez-Roselloj,P. Barrio, A. SimonFuentes, "From 2000 to mid - 2010: a fruitful decade for the synthesis of pyrazoles", Chem. Rev, vol. 111, pp. 6984-7034, 2011.

9. K.S Gudmundsson, B.A Johns, S.H Allen, "Pyrazolopyridines with potent activity against herpesviruses: effects of C5 substituents on antiviral activity", Bioorg Med Chem Lett, vol. 18, pp. 1157-1161, 2008.

10. D.B Kendre, R.B Toche, M.N Jachak, "Synthesis of novel dipyrazolo[3,4-b:3,4-d] pyridines and study of their fluorescence behavior", Tetrahedron, vol. 63, pp. 11000-11004, 2007.

11. A. Bazgira, M.M Khanaposhtani, A.A Soorki, "One pot synthesis and antibacterial activities of pyrazolo[4',3': 5,6] pyrido[2,3-d]pyrimidine dione derivatives", Bioorg Med Chem Lett, vol. 18 pp. 5800-5803, 2008.

12. R.S Varma, "Solvent-free organic syntheses. Using supported reagents and microwave irradiation", Green Chem., vol. 1, pp. 43-55, 1999.
13. B.M Trost, M.K Brennan, "Asymmetric Syntheses of Oxindole and Indole Spirocyclic Alkaloid Natural Products”, Synthesis, vol. 18, pp. 3003-3025, 2009.

14. R. Ranjith Kumar,S. Perumal,P. Senthilkumar, P. Yogeeswari, D.A Sriram, "A facile synthesis and antimyobacterial evaluation of novel spiro-pyrido-pyrrolizines and pyrrolidines", Eur J Med Chem, vol. 44, pp. 3821-3829, 2009.

15. 15. Y.S Wu, J. Cai, Z.Y Hu, G.H Lin, "A new class of metal-free catalysts for direct diastereo- and regioselective Mannich reactions in aqueous media", Tetrahedron Lett, vol. 45, pp. 8949-8952, 2004.

16. 16. J. Yanga, X. Xua, J. Rui, Z. Wang, Y. Zhang, S. Wang, L. Wu, "Synthesis, optical properties and application of a set of novel pyrazole nopinone derivative", Spectrochimica Acta Part A: Molecular and BiomolecularSpectroscopy, vol. 183, pp. 60-67, 2017.

17. F. López-Muñoz, R. Ucha-Udabe, C. Alamo, "The history of barbiturates a century after their clinical introduction", Neuropsychiatric Disease and Treatment, vol. 1, pp. 329-343, 2005.

18. N. Moussier, L. Bruche, F. Viani, M. Zanda, "Fluorinated Barbituric Acid Derivatives: Synthesis and Bioactivity", Curr. Org. Chem, vol. 7, pp. 1071-1080, 2003.

19. A. M. Al-Majid, A. Barakat, H. J. Al-Najjar, Y. N. Mabkhot, H. A. Ghabbour, H. K. Fun, "Tandem Aldol- Michael Reactions in Aqueous Diethylamine Medium: A Greener and Efficient Approach to Bis-Pyrimidine Derivatives", Int. J. Mol. Sci, vol. 14, pp 23762-23773, 2013.

20. J. T. Bojarski, J. L. Mokrosz, H. J. Bartoń, M. H. Paluchowska, “ Recent progress in barbituric acid chemistry", Advances in heterocyclic chemistry, vol. 38, pp. 229-297, 1985.

21. S. R. Guillén, C. M.Guzmán, "Historical aspects and applications of barbituric acid derivatives. A review", Die Pharmazie, vol. 43, pp. 827-829, 1988.

22. J. B. Taylor Modern Medical Chemistry; Prentice Hall: New York, NY, USA, 1994

23. A. Barakat, H. J. Al-Najjar, A. M. Al-Majid, S. M. Soliman, Y. N. Mabkhot, M. R. Shaik, H. A. Ghabbour, H. K. Fun, "Synthesis, NMR, FT-IR, X-ray structural characterization, DFT analysis and isomerism aspects of 5- (2,6-dichlorobenzylidene) pyrimidine-2,4,6 $(1 \mathrm{H}, 3 \mathrm{H}, 5 \mathrm{H})-$ trione", Spectrochim. Acta Mol. Biomol. Spectrosc, vol 147, pp. 107-116, 2015.

24. A. Barakat, A. M. Al-Majid, H. J. Al-Najjar, Y. N. Mabkhot, S. Javaid, S. Yousuf, M. I. Choudhary, "Zwitterionic pyrimidinium adducts as antioxidants with therapeutic potential as nitric oxide scavenger", Eur. J. Med. Chem, vol. 84, pp. 146-154, 2014.

25. A. Barakat, S. M. Soliman, A. M. Al-Majid, G. Lotfy, H. A. Ghabbour, H. K. Fun, S. Yousuf, M. I. Choudhary, A. Wadood, "Synthesis and structure investigation of novel pyrimidine-2,4,6 trione derivatives of highly potential biological activity as anti-diabetic agent”, J. Mol. Stru, vol. 1098, pp. 365-376, 2015.

26. A. Barakat, M.S. Islam, A. M. Al-Majid, H. A. Ghabbour, H. K. Fun, K. Javed, R. Imad, S Yousuf, M. I. Choudhary, A. Wadood, “ Synthesis, invitro biological activities and in silico study of dihydropyrimidines derivatives", Bioorg. Med.Chem, vol. 23, pp 6740-6748, 2015.

27. B. B. Ivanova, M. Spiteller, "Possible Application of the Organic Barbiturates as NLO materials", Crystal Growth \& Design, vol. 10, pp. 2470-2474, 2010.

28. A. D. Azzahari, V. Selvanathan, M. Rizwan, F. Sonsudin, R. Yahya, “ Conductivity or rheology? Tradeoff for competing properties in the fabricaton of a gel polymer electrolyte based on chitosan-barbiturate derivative", Ionics, vol. 24, pp. 3015-3025, 2018.

29. S. Bansal, M. Bala, S. K. Suthar, S. Choudhary, S. Bhattacharya, V. Bhardwaj, S. Singla, A. Joseph, "Design and synthesis of novel 2-phenyl-5-(1,3-diphenyl-1H-pyrazol-4-yl)-1,3,4-oxadiazoles as selective COX-2 inhibitors with potent anti-inflammatory activity", Eur. J. Med. Chem, vol. 80, pp. 167-174, 2014.

30. C. Peng, P.Y. Ayala, H. B. Schlegel, M. J. Frisch, "Using redundant internal coordinates to optimize equilibrium geometries and transistion states", J. Comput. Chem, vol. 17, pp. 49-56, 1996.

31. M. J. Frisch, G. W. Trucks, H. B. Schlegel, G. E. Scuseria, M. A. Robb, J. R.Cheeseman, et. al., "Gaussian 09 (Gaussian Inc., Wallingford CT)", Revision D, 1, 2009

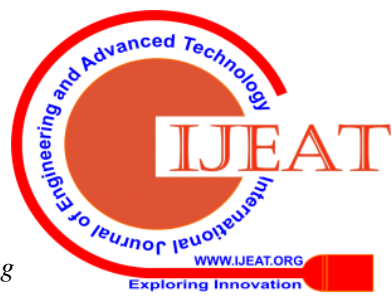




\section{Synthesis and Density Functional Theory Analysis of Pyrazole Integrated Pyrimidinetrione}

32. M. Dinesh Raja, S. Arulmozhi, J. Madhavan, "UV-Vis, HOMO-LUMO and hyperpolarizability of L-Phenylalanine L-Phenylalaninium-Benzoic acid”, International Journal of Scientific and Engineering Research, vol. 5, pp. 15-17, 2014.

\section{AUTHORS PROFILE}

Shunmugam Iniyaval obtained her Bachelor of Science degree in Chemistry from Sri Parasakthi College for Women, Courtallam in 2013. She earned her Master's degree in Chemistry in 2015 from Sri Paramakalyani College, Alwarkuruchi. She is currently a PhD student at Kalasalingam Academy of Research and Education, Krishnankoil. She has been working in the area of heterocyclic synthesis and medicinal chemistry.

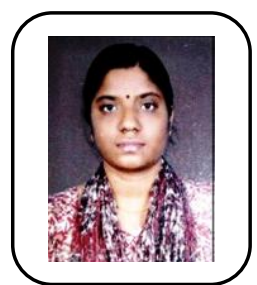

Krishnaraj Padmavathy received her Bachelor of Science degree in Chemistry from Bharathidasan college for women, Pondicherry in 2004. She obtained her Master's degree from Kanchi Mamunivar Centre for Postgraduate Studies, Pondicherry in 2006. She is currently a PhD student at Kalasalingam Academy of Research and Education, Krishnankoil, India. Her focus of research is methodology development and heterocyclic construction for biological applications.

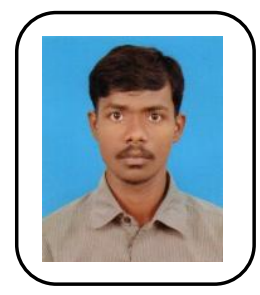

Ramar Sivaramakarthikeyan earned his BSc degree in Chemistry from VHNSN College, Virudhunagar, affiliated to Madurai Kamaraj University, Tamilnadu, India in 2013. He completed his MSc in Chemistry in 2015 from Kalasalingam Academy of Research and Education, Krishnankoil. Currently he is pursuing $\mathrm{PhD}$ at Kalasalingam Academy of Research and Education, Krishnankoil. $\mathrm{He}$ has been working in the area of biopertinent heterocycles.

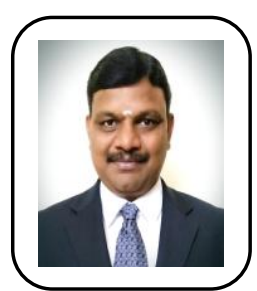

Dr. Chennan Ramalingan is serving as Professor of Chemistry at KARE. He received his $\mathrm{PhD}$ in Organic Chemistry from Annamalai University (2002). After he served as Research Professor at Kyungpook National University, South Korea followed by Assistant Professor at Osaka University, Japan (8 years), he joined at Kalasalingam Academy of Research and Education, Krishnankoil (2011). His research interests include organic synthesis, medicinal chemistry and material chemistry. He published more than 70 International research articles. 